\title{
Opiatabhångigkeit
}

\section{Substitution kann vor Überdosis schützen}

\begin{abstract}
- Die Anzahl der Drogentoten in Deutschland steigt kontinuierlich an, berichtete Stephan Walcher, niedergelassener Suchtmediziner in München. Probleme bereite eine „nahezu unübersehbare Flut" neuer psychoaktiver Substanzen (NPS), die oft nur schwer zu identifizieren sind. Alarmierend sei außerdem, dass ärztlich verordnete starke Schmerzmittel wie Opioide inzwischen für mehr Drogentote verantwortlich sind als alle illegalen Drogen zusammen. Zunehmender Beliebtheit erfreue sich auch Pregabalin als Ersatzdroge für Benzodiazepine.
\end{abstract}

\section{Wechselwirkungen beachten}

Vorrangiges Ziel in der Therapie von Drogenabhängigen sei die Sicherung des
Überlebens. Deshalb habe die vor mehr als 20 Jahren eingeführte orale Substitutionstherapie (OST) einen hohen Stellenwert. „Das Sterberisiko unter Substitution beträgt nur etwa ein Viertel der Szenemortalität", erklärte Walcher.

Um medizinische Notfälle zu vermeiden, sollten mögliche Arzneimittelinteraktionen berücksichtigt werden. So können Komedikamente oder Zusatzdrogen, die ebenfalls über das Cytochrom-P450System verstoffwechselt werden, zu toxischen Plasmaspiegeln und gefährlichen Herzrhythmusstörungen führen. Walcher riet deshalb dringend zum EKGMonitoring vor und nach der OST-Einstellungsphase, um eine QT-Zeit-Verlängerung auszuschließen.

\section{Halsbeschwerden}

\section{Deutliche Unterschiede bei Mucilaginosa}

Bei Heiserkeit und Halskratzen sind Hyaluronsäure-haltige Präparate eine Therapieoption. Dass die Präparate unterschiedlich gut wirken, zeigte eine prospektive Beobachtungsstudie. Bakterielle Infektionen wurden ausgeschlossen. Die Patienten erhielten entweder GeloRevoice ${ }^{\bullet}$ oder eine von drei anderen Halstabletten dieser Kategorie. Es erfolgte eine Bewertung der Symptome durch Arzt und Patient anhand eines Scores (1-10 Punkte) zu Beginn und am Ende der Therapie.

Die Ergebnisse: Lag das Beschwerdeausmaß zu Beginn zwischen 3,5 und 4,3 Punkten, verringerte es sich in allen vier Gruppen, berichtete Prof. Hans Behrbohm, Berlin. Bei den Vergleichspräparaten lag der Wert am Ende zwischen 1,9 und 2,1 Punkten, unter GeloRevoice ${ }^{\circ}$ bei nur 0,9 (Ausgangswert: 4,3). „Die Re- duktion aller Symptome gelang mit dem Testpräparat bei fast $80 \%$ der Patienten", so der HNO-Facharzt. „Bei den Vergleichspräparaten war das nur bei rund 40-48\% der Patienten der Fall." Unter dem Testpräparat waren zudem am dritten Tag 40\% Patienten beschwerdefrei, mit den Vergleichspräparaten nur rund $10-15 \%$. Behrbohm führt das auf die lange Verweildauer des Schutzfilms durch das Hydro-Depot ${ }^{\circledR}$ zurück. So seien die Schleimhäute besser und länger geschützt.

GeloRevoice ist für Erwachsene und Kinder ab sechs Jahren zugelassen. Es ist in den Geschmacksrichtungen KirschMenthol, Cassis-Menthol und Grapefruit-Menthol erhältlich. Bis zu sechs Tabletten täglich können gelutscht werden, vorzugsweise nach dem Essen. „Das verhindert die Beschädigung des Schutz-
Wie gut Substitution funktionieren kann, zeigt eine Studie aus Frankreich [1]. Nach Einführung von Buprenorphin und dem verstärkten Einsatz von Methadon ging die Anzahl der Drogentoten auf ein Sechstel zurück.

Durch die starke Rezeptorbindung verdrängen Ersatzdrogen das andere Opiat und schützen dadurch vor einer Überdosierung. Das am häufigsten eingesetzte Substitut ist Methadon (z. B. Methaddict ${ }^{\circ}$, Methaliq $\left.^{\circ}\right)$, gefolgt von Levomethadon (z. B L-Polaflux ${ }^{\circ}$ und Buprenorphin (z. B. Buprenaddict ${ }^{\circ}$ ).

\section{Dr. Martina-Jasmin Utzt}

- Symposium "Substitution -Interaktion mit Medikamenten und Drogen", 17. Interdisziplinärer Kongress für Suchtmedizin; München, Juli 2016 (Veranstalter: Hexal)

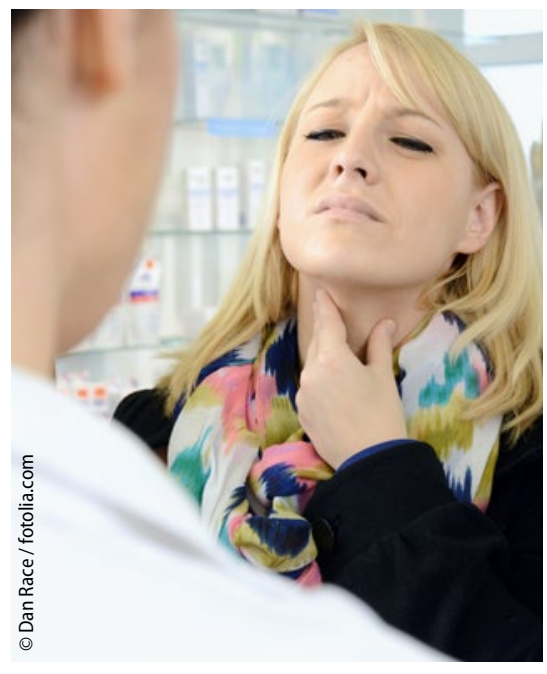

Hyaluronsäure hilft nicht nur am Gelenk.

films durch Nahrungsbestandteile“, erklärte Behrbohm.

\section{Dr. Michael Hubert}

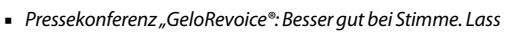
Deine Stimme klingen wie 10.000!"; Hamburg, August 2016 (Veranstalter:Pohl-Boskamp) 